\title{
A preliminary study on occurrence and characterization of extended spectrum beta lactamase producing extra intestinal pathogenic Escherichia coli isolated from dogs
}

WAPM Dewasmika, DRA Dissanayake

\section{Introduction}

Extra intestinal pathogenic Escherichia coli (ExPEC) producing extended spectrum $\beta$-lactamase (ESBL) and resistant to fluoroquinolones has emerged and disseminated globally. ESBL producing organisms are resistant to third and fourth generation cephalosporins and monobactams and may also express $A m p C \beta$-lactamases and aminoglycoside resistance. ESBLs confer the resistance through enzymes such CTX-M, TEM and SHV. Although the prevalence of ESBL producing organisms among companion animals is not well studied, it is clearly increasing. This paper presents a preliminary study on occurrence of ESBL producing ExPEC among dogs in Sri Lanka and the rates of selected genes encoding resistance.

\section{Methods}

ExPEC isolates $(n=15)$ collected from dogs were subjected to antimicrobial susceptibility testing $(\mathrm{AST})$ with 15 antimicrobial drugs $\left(1^{\text {st }}, 2^{\text {nd }}, 3^{\text {rd }}\right.$ and $4^{\text {th }}$ gen cephalosporins, quinolones, betalactams and monobactams, sulpha trimethoprim and amikacin) following EUCAST guidelines. Organisms showing resistance to 3rd generation cephalosporins were presumptively identified as ESBL and were confirmed by double disk synergy test. Presence of genes encoding beta lactamases (bla $\mathrm{TEM}_{\mathrm{T}}$, blashv, bla $\mathrm{C}_{\mathrm{CT}-\mathrm{M})}$ and quinolone resistance (qnrmA, qnrmB and qnrmS) were assessed by PCR.

\section{Results}

Of the 15 isolates tested, 12 showed resistance to $2^{\text {nd }}, 3^{\text {rd }}$ or $4^{\text {th }}$ generation cephalosporins and $4 / 12$ isolates were confirmed as ESBLs by double disk synergy test. Six isolates which showed resistance to all $3^{\text {rd }}$ generation cephalosporins and three isolates with ceftriaxone and/or cefpodoxime resistance contained Blaтем, and BlactX-м genes. Blashv was detected only in three isolates. All fifteen isolates were resistant to ampicillin and 13 were resistant to Amoxy+clavulonated. Though only four isolates were resistant to quinolones, the genes qnrmS, $q n r m \mathrm{~B}$ and qnrmA were found in 15, 8 and 3 isolates respectively.

\section{Conclusion}

ExPEC with ESBL and quinolone resistance were detected in dogs and warrant further studies to determine whether they belong to the globally disseminated ST131 clone.

Keywords: Escherichia coli, extended spectrum beta lactamase, extra intestinal

Funding: NRC research grant No: 19-089

Department of Department of Veterinary Clinical Sciences, Faculty of Veterinary Medicine and Animal Science, University of Peradeniya, Sri Lanka 